\title{
L-Carnitine affects preimplantation embryo development toward infertility in mice
}

\author{
Christiana Kyvelidou", Dimitris Sotiriou, ${ }^{1, *}$, Tania Antonopoulou, ${ }^{1,}$, Margarita Tsagkaraki $^{2,3}$, \\ George J Tserevelakis ${ }^{2}$, George Filippidis ${ }^{2}$ and Irene Athanassakis ${ }^{1}$ \\ ${ }^{1}$ Department of Biology, University of Crete, Crete, Greece, ${ }^{2}$ Institute of Electronic Structure and Laser, Foundation \\ of Research and Technology, Crete, Greece, and ${ }^{3}$ Department of Physics, University of Crete, Crete, Greece \\ Correspondence should be addressed to I Athanassakis; Email: athan@biology.uoc.gr
}

*(D Sotiriou and T Antonopoulou contributed equally to this work)

\begin{abstract}
L-Carnitine ( $\mathrm{L}-\mathrm{Cn}$ ), despite the beneficial role as energy-generating substance delivering long-chain fatty acids to the $\beta$-oxidation pathway in mitochondria, has been accused to cause an endometriosis-like state to BALB/c mice manifested by increased inflammatory cytokines in serum and peritoneal fluid, accumulation of immune cells in the peritoneal cavity and uterine walls and most importantly, correlating to infertility. Exploring this type of infertility, the effect of L-Cn on preimplantation embryo development, ovarian integrity and systemic maternal immunity was studied. Using nonlinear microscopy analysis, which was shown to be a powerful tool for determining embryo quality by quantitatively estimating the lipid body (LB) content of the cells, it was shown that in vitro and in vivo administration of L-Cn significantly decreased LB mean area in zygotes. Daily intraperitoneal administration of $2.5 \mathrm{mg}$ L-Cn for 3, 4 and 7 days to mice significantly decreased the percent of normal zygotes. However, only the 7-day treatment persisted by affecting 2- and 8-cell stage embryos, while almost abolishing blastocyst development. Such effects were accompanied by abnormal ovarian histology, showing increased numbers of corpora luteus and elevated progesterone concentration in the serum. In addition, it was shown that the 7-day L-Cn treatment pushed maternal systemic immunity toward inflammation and immunosuppression by increasing CD11b-, CD25- and CD11bGr1-positive cells in spleen, which opposed the necessity for immunostimulation at these early stages of pregnancy. In conclusion, the results presented here demonstrated that elevated doses of L-Cn affect early stages of embryo development, leading to infertility.

Reproduction (2016) 152 283-291
\end{abstract}

\section{Introduction}

L-Carnitine (L-Cn) is an essential energy-providing compound to the cell delivering long-chain fatty acids to the $\beta$-oxidation pathway in the mitochondrial matrix for catabolism. Upon coupling to coenzyme-SH, acylCoA follows the L-Cn transporter system, where the acylgroup is transferred to the hydroxyl group of L-Cn to form the acyl-carnitine and cross the inner mitochondrial membrane. After transferring the acyl group to coenzyme $\mathrm{A}(\mathrm{CoA}), \mathrm{L}-\mathrm{Cn}$ returns to the mitochondrial intramembrane space, while the acyl residue as acyl-CoA thioester enters the $\beta$-oxidation pathway or serves in triglyceride biosynthesis (Michal 1999). In some cases, a portion of the semifinal product of fatty acid $\beta$-oxidation, the acetoacetyl-CoA, is used for the formation of cholesterol and $\beta$-OH-butyrate (ketone bodies) supplying energy as shown for brain and muscle cells (Michal 1999).

Any damage in the L-carnitine transporter system does not allow oxidation of long-chain fatty acids, leading to primary or secondary carnitine deficiencies such as myopathies, systemically progressive cardiopathies or organic acidurias, respectively (Karpati et al. 1975). To this extend, L-Cn has been used as an additive to various therapeutic regimens against HIV infection, tuberculosis, myopathies, cardiopathies, neuropathies as well as to baby foods and milk (Famularo et al. 1977). L-Carnitine is often used to improve sperm quality $(\mathrm{Ng}$ et al. 2004), athletic performance and weight loss (Brass 2004). Studies on the effect of L-Cn on the immune system have been producing contradictory results. Although immune cells consume a small portion of L-Cn, it was shown to increase the arachidonic acid-induced production of prostaglandin E1 (PGE1) and PGE2 by macrophages and decrease $\beta-\mathrm{OH}$-butyrate production (Athanassakis et al. 2003). L-Carnitine administration to mice has been shown to affect the humoral and cellular immune responses by decreasing interleukin-2 (IL-2) production in response to specific antigenic stimulus (Athanassakis et al. 2001). In addition, exogenously provided L-carnitine mobilizes fatty acid reserves from the immune cells and alters their energetic state, which 
can thereafter influence various pathways affecting cell maturation and changes in cytokine production, events that determine the maintenance of homeostasis to the organism (Athanassakis et al. 2003).

L-Carnitine administration to female mice has been shown to induce an endometriosis-like state, which is accompanied by significant increase in inflammatory cytokines in serum and peritoneal fluid, accumulation of immune cells in the peritoneal cavity and uterine walls and most importantly, infertility (Dionyssopoulou et al. 2005). In those studies, it was shown that when receiving intraperitoneal injections of $2.5 \mathrm{mg} \mathrm{L-Cn}$ per day for 7 days, females could reach estrus, copulate with males and, despite the vaginal plug, they failed to further proceed to pregnancy.

In order to exploit the L-Cn-induced infertility state, the present study was designed to evaluate oocyte and preimplantation embryo quality, if any. Previous studies from our laboratory have established a noninvasive imaging technology evaluating preimplantation embryo quality using a third harmonic generation (THG) nonlinear microscopic technique (Kyvelidou et al. 2011). According to this technology, when intense laser radiation interacts with matter, there is a finite probability that three photons of the fundamental angular frequency $\omega$ will be destroyed and a new photon of angular frequency $3 \omega$ will be simultaneously created in a single-quantum mechanical process (Barad et al. 1997). This nonlinear coherent scattering event is known to be sensitive to local differences as to the refractive index and the thirdorder susceptibility values of the interacting medium, as well as its respective dispersion properties (Debarre \& Beaurepaire 2007). According to the current literature, the most prominent subcellular contributors of strong THG signals are mitochondria (Hsieh et al. 2008) and lipid bodies (Débarre et al. 2006, Watanabe et al. 2010). Such technology allowed extracting valuable quantified information as to the energetic status of preimplantation embryos, time evolution of different developmental stages, embryo polarization before mitotic division and blastomere equivalence (Kyvelidou et al. 2011).

Thus, taking advantage of THG imaging, it was possible to evaluate preimplantation embryo health and follow the effect of L-Cn on embryo growth in vitro as well as in vivo, while parallel evaluation of the ovarian tissue as well as the maternal immune state could allow localization of the L-Cn-induced fertility defect and provide an additional cause to infertility.

\section{Materials and methods}

\section{Animals}

$\mathrm{BALB} / \mathrm{c}$ mice (Mus musculus) were purchased from Charles River (Milan, Italy), maintained in the animal facility at the University of Crete (Crete, Greece) and approved according to national guidelines (EL91-BIObr-09). Six- to fourteen-week-old male and female mice $(20 \pm 0.3 \mathrm{~g})$ were used in all experiments.

\section{Administration of substances and mating}

$\mathrm{BALB} / \mathrm{C}$ female mice were superovulated by intraperitoneal injections of pregnant mare serum (PMSG, $5 \mathrm{IU} / \mathrm{mice}$, Sigma, G4877) and human chorionic gonadotropin (hCG, 5IU/ mice, Sigma, CG5-1VL) at an interval of $47 \mathrm{~h}$. The time of administration of gonadotropins was based on the light cycle used. L-Carnitine (L-carnitine inner salt, $2.5 \mathrm{mg} / \mathrm{mouse}$, Sigma, C0158) was administered by intraperitoneal injection daily for $3,4,5$ or 7 days before the scheduled superovulation and mating. The selected daily dose (Dionysopoulou et al. 2001) corresponds to $1 / 3$ of the daily dose administered in humans to increase athletic performance or sperm quality $(2-6 \mathrm{~g} /$ day; total body content of $\mathrm{L}-\mathrm{Cn}$ is about $20 \mathrm{~g}$ in a man weighing 155 pounds; Brass 2004). Superovulated females were placed with male breeders $2 \mathrm{~h}$ post-hCG injection and remained caged together overnight. Next morning, mating was detected by the presence of a vaginal plug. The females used in each set of experiments were randomly chosen from the same breed.

\section{Embryo harvesting}

Depending on the desired embryo stage, plug-positive females were killed at $18 \mathrm{~h}$ post-hCG (Day 0, zygotes), $44 \mathrm{~h}$ post-hCG (Day 1, 2-cell embryos), $72 \mathrm{~h}$ post-hCG (Day 3, 8-cell embryos) or $96 \mathrm{~h}$ post-hCG (Day 4, blastocysts) injection.

\section{Embryo cultures}

Zygotes from control superovulated plug-positive females were harvested and cultured in $50 \mu \mathrm{L}$ droplets of prewarmed mHTF (Kito et al. 2004) medium with or without $400 \mu \mathrm{M} \mathrm{L-Cn}$ (Athanassakis et al. 2001, 2003) (with maximum 10 embryos per drop) covered with mineral oil (Sigma, M8410), at $37^{\circ} \mathrm{C}$ in a $5 \% \mathrm{CO}_{2}$ atmosphere. The embryos were cultured for $24 \mathrm{~h}$ to assess their ability to overcome the '2-cell block' and undergo their first cleavage.

\section{Third harmonic generation, TPEF and confocal imaging}

For THG imaging, harvested embryos were washed in PBSBSA $0.1 \% \mathrm{w} / \mathrm{v}$ (Sigma P4417; Sigma, A7906), fixed with PFA $2 \% \mathrm{w} / \mathrm{v}$ (Sigma, 158127) and placed in PBS-BSA $0.1 \% \mathrm{w} / \mathrm{v}$ between two very thin $(\sim 70 \mu \mathrm{m})$ round glass slides (Marienfeld). The glass slides were separated with a $100 \mu \mathrm{m}$ thick spacer in order to avoid damaging the samples. The process of the THG signal detection was performed as described previously (Kyvelidou et al. 2011). Briefly, a femtosecond laser beam was guided into a modified Nikon upright microscope (Eclipse ME600D) and subsequently focused tightly into the sample through an objective lens (C-Achroplan, Carl Zeiss; 32X, NA 0.85 , water immersion). A pair of galvanometric mirrors was used for the lateral scanning, whereas a motorized translation Z-stage was employed for the focal plane selection. Following the filtering with a $340 \mathrm{~nm}$ color glass filter, THG signals were detected in the forward direction by a photomultiplier tube 
(H9305-04, Hamamatsu). The average laser power on the sample plane was $20 \mathrm{~mW}(0.4 \mathrm{~nJ}$ per pulse). Every recorded slice image $(500 \times 500$ pixels $)$ was the result of 30 averaged scans, each of them recorded in less than $1 \mathrm{~s}$.

Two photon excitation fluorescence (TPEF) signals for nile red and mitotracker detection were recorded in the backward direction using another photomultiplier tube (R4220 Hamamatsu). A short-pass filter (SPF $650 \mathrm{~nm}$ CVI) was placed at the photomultiplier input to cut off the reflected laser light. By detecting THG images in the forward direction and TPEF images in the backward direction, the experimental apparatus allowed the performance of co-localization measurements.

For TPEF imaging and THG signal co-localization, embryos were washed in PBS-BSA $0.1 \% \mathrm{w} / \mathrm{v}$, fixed with PFA $4 \% \mathrm{w} / \mathrm{v}$ (Sigma, 158127), permeabilized with PBS-Triton X-100 0.025\% v/v (Sigma, X100) and stained with Nile Red $(10 \mu \mathrm{g} / \mathrm{mL}$, Sigma, N3013). For mitochondrial staining, fresh embryos were incubated with Mitotracker Red (250 nM, Molecular Probes, $\mathrm{M}-7512$ ) at $37^{\circ} \mathrm{C}$ for $30 \mathrm{~min}$. In other experiments, embryos were stained with Nile red and analyzed using Leica TCS SP confocal microscope and LCS software (Leica Microsystems). In such case, all samples were mounted (Vectashield, Vector, $\mathrm{H}-1000)$ and observed within $24 \mathrm{~h}$.

\section{THG signal quantification}

The quantification of THG signals consisted of two distinct image processing methodologies. The first included the determination of the total number of particles and their respective average size. Following this type of processing, a threshold of the normalized slice images was applied, using a global intensity threshold value. The resulting binary images were further processed through the 'Analyze particles' built-in function of the Java-based software ImageJ, whereas the statistical analysis (mean values calculation/t-tests) of the acquired data was performed through MATLAB.

The second image processing calculated the mean THG intensity for each measured specimen. To this extend, a customdesigned algorithm, programmed in MATLAB environment was used. The algorithm loaded the normalized Z-stack of images and delimited automatically the representative embryo region for each slice through proximity of intensity selection function for a fixed tolerance level. The selected pixel values served as input for the calculation of the embryo's mean THG intensity, the variability of the signals and the statistical hypothesis testing between different sample groups.

\section{Ovary harvesting and histochemistry}

Ovaries were harvested approximately 18 h post-hCG injections, when 1-cell embryos were collected from control, 5- and 7-day L-Cn-treated superovulated mice. Tissues were washed with PBS, fixed overnight with PBS-PFA $4 \% \mathrm{w} / \mathrm{v}$ at $4{ }^{\circ} \mathrm{C}$, rinsed in $\mathrm{PBS}$ and $\mathrm{NaCl} 0.9 \% \mathrm{w} / \mathrm{v}$ at room temperature, dehydrated using decreasing concentrations of ethanol (Riedel-de Haën, 32221) and xylene (Sigma, 534056), embedded overnight in paraffin (Sigma, P3683) at $63^{\circ} \mathrm{C}$ and were finally let to cool at $4^{\circ} \mathrm{C}$. The tissues were cut in $7 \mu \mathrm{m}$ slices on a microtome (Leica RM2125 RTS). Sections were deparaffinized in xylene and rehydrated in decreasing concentrations of ethanol. Sections were stained with hematoxylin-eosin (Mayer's Haemalum and eosin $Y 2 \% \mathrm{w} / \mathrm{v}$ ), dehydrated in ethanol and xylene and mounted for histological evaluations. Each section was observed and analyzed by light stereomicroscope (Leica M28). Ten central sections were selected and their total area was measured and averaged using ImageJ.

\section{Evaluation of progesterone levels in serum}

Blood was collected by cardiac puncture under anesthesia from pregnant mice $18 \mathrm{~h}$ post-hCG administration, submitted or not to the 7-day L-Cn treatment. Blood was left to clot at room temperature for $30 \mathrm{~min}$ and serum was collected after spin down. Serum was kept at $-20^{\circ} \mathrm{C}$ for short periods of time and at $-80^{\circ} \mathrm{C}$ for long-term maintenance. Progesterone levels were detected using the DRG Progesterone ELISA kit (DRG International, Inc., New Jersey, USA; EIA-1561, kindly provided by Prof. M. Pavlidis, Department of Biology, University of Crete, Greece). The results were calculated automatically using a $4 \mathrm{PL}$ (4 Parameter Logistics) curve fit performed in GraphPad Prism 6.01 (Graphpad Software).

\section{Spleen cell isolation, immunofluorescence and flow cytometry}

Spleen cells were harvested in Hank's balanced salt solution (HBSS, Gibco, 14170) by gently squeezing and washed with PBS. Cells were fixed with PBS-PFA $4 \% \mathrm{w} / \mathrm{v}$ on ice and blocked with PBS-BSA $2 \% \mathrm{w} / \mathrm{v}$. The monoclonal anti-mouse antibodies anti-CD11b PE (EuroBioSciences, M22127P), anti-CD25 PE (EuroBioSciences, M22128P), anti-IA FITC (BD Biosciences, 553547), anti-CD19 PE (Immunotools, 22220194), anti-CD4 PE (Immunotools, 22150044), anti-CD8 PE (Immunotools, 22150084) and anti-Gr-1 FITC (Immunotools, 22155243) were used at the concentration of $1 \mu \mathrm{g} / \mathrm{mL}$. Fluorescent intensity was evaluated by flow cytometry (FACScan, Becton Dickinson) and the results were analyzed using the FCS Express V3 software (De Novo Software).

\section{Statistical analysis}

Data were analyzed with two-tailed paired or unpaired Student's $t$-test. $P$ values $<0.05$ were considered significant $(*)$, values $<0.01$ were considered very significant $\left(^{* *}\right)$ and values $<0.001$ and $<0.0001$ were considered highly significant $(* * *$ and $* * * *)$. Statistics were performed using GraphPad Prism 6.01 (GraphPad Software).

\section{Results}

Previous studies have correlated embryo quality to lipid body (LB) quantity, quality and distribution to embryo health and development (Kyvelidou et al. 2011). In addition, other studies from our laboratory had shown that long-chain fatty acid oxidation cofactor, L-Cn, could abrogate the reproductive ability of female mice (Dionyssopoulou et al. 2005). Thus, the L-Cn-induced infertility could lie on embryo quality defects, due to 
increased lipid catabolism. Taking these observations together, it was inquired whether the effect of L-Cn is limited to embryo quality, or whether it affects different reproductive processes.

\section{Effect of L-Cn on embryo development}

As an initial attempt, zygotes were isolated from control superovulated mice, randomly divided into two groups and cultured in the presence or not of $400 \mu \mathrm{M}$ L-Cn. Embryo development and LB quantity and distribution were examined $24 \mathrm{~h}$ after culture initiation (Fig. 1). In the presence of $\mathrm{L}-\mathrm{Cn}, 30 \%$ less zygotes $(P=0.0325)$ reached the stage of 2-cells (Fig. 1A). The distribution of LBs was examined by either confocal microscopy upon staining with nile red or THG imaging (Fig. 1C). Both approaches showed a significant reduction in the LB content of L-Cntreated embryos. Quantification of the THG signal using a MATLAB-developed algorithm (see "Methods" section) showed a $39 \%$ reduction in mean LB area in the L-Cntreated embryos as compared with controls $(P=0.0075$; Fig. 1B). Indeed, in preimplantation embryos, THG signal co-localized with nile red and not mitotracker red staining (visualized via TPEF measurements) (Fig. 2) indicating that THG quantification directly correlates to LB content in the embryos.

A
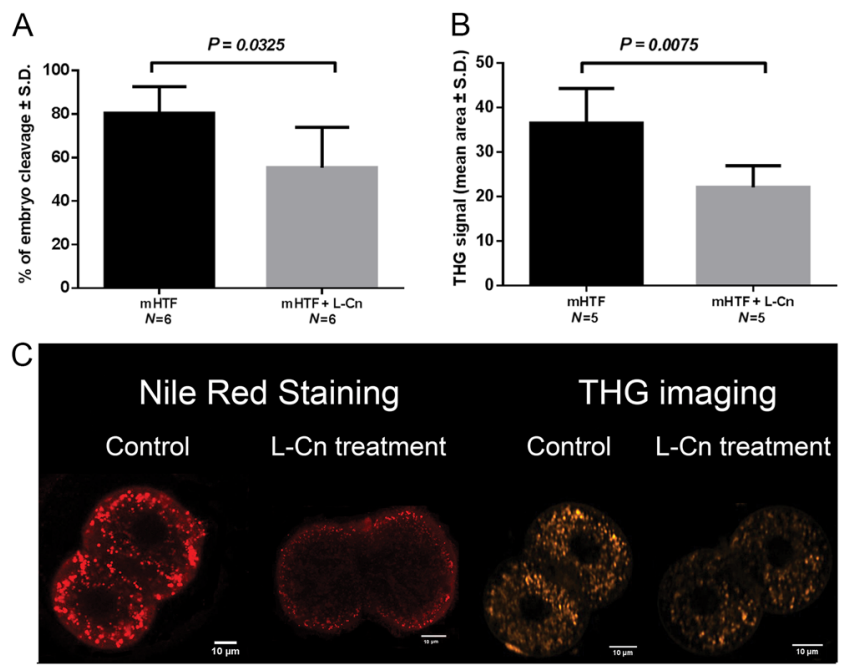

Figure 1 In vitro effect of L-Cn on preimplantation embryo development. Zygotes harvested from superovulated BALB/C mice were randomly divided into two groups and incubated with or without $400 \mu \mathrm{ML}-\mathrm{Cn}$ for $24 \mathrm{~h}$ at $37^{\circ} \mathrm{C}$ in a $5 \% \mathrm{CO}_{2}$ atmosphere. The percent of embryo cleavage $(\mathrm{A})$ and THG mean area $(B)$ were calculated as described in Methods. The results in $(A)$ represent the mean of 6 experiments ( \pm S.D.) with a total number of 109 and 114 embryos tested in control (mHTF) and L-Cn $(\mathrm{mHTF}+\mathrm{L}-\mathrm{Cn})$ groups, respectively. The results in $(\mathrm{B})$ represent the mean of five embryos in each case ( \pm S.D.). $P$ values were calculated using the GraphPad software statistical analysis tools (paired $t$-test in $(A)$, unpaired $t$-test in (B)). Upon culture termination, embryos were either stained with Nile red and visualized using confocal microscopy or processed to THG imaging techniques (C). Scale bars are shown in all cases.
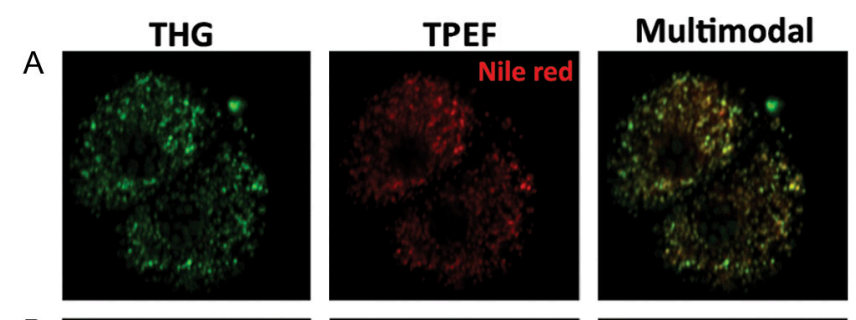

B
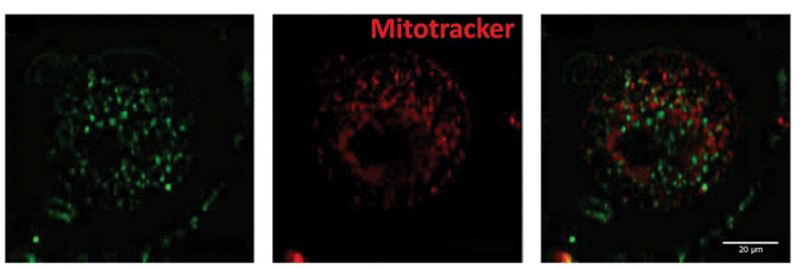

Figure 2 Co-localization of THG and Nile red signal in two-cell stage embryos. Embryos were freshly isolated 44 or $18 \mathrm{~h}$ post-hCG administration, manipulated as described in Methods, stained with Nile red (A) or mitotracker (B) and processed to TPEF (red) and THG imaging (green) by employing the set up described in methods.

In order to evaluate whether L-Cn could also affect embryo quality in vivo, superovulated females received a 7-day L-Cn treatment, which was previously shown to affect fertility and induce an endometriosis-like state to the mother. Zygotes were isolated from superovulated control or superovulated L-Cn-treated females, and submitted to THG imaging (Fig. 3A). Although THG signal mean intensity did not differ between zygotes isolated from control and treated animals (Fig. 3B), there was a significant reduction of $39 \%$ in the number of LB content of the zygotes ( $P=0.0079$; Fig. $3 \mathrm{C})$ and a reduction of $21 \%$ in the mean area of $\operatorname{LBs}(P=0.0489$; Fig. 3D). In this case, however, LBs showed a different distribution as compared with controls, while they seemed to coagulate, forming big clusters/depositions (Fig. 3E).

These results indicated that although L-Cn, by exerting a direct effect on embryos in vitro, reduced LB total area, when administered in vivo, additional events took place, which disturbed LB performance and consequently embryo development.

\section{Fertilization and embryo growth upon L-Cn treatment}

In order to define the lowest dose of L-Cn that could affect embryo development, superovulated females received the L-Cn treatment for 3, 4, 5 or 7 days and embryo development was assessed on days 0 and 1 of pregnancy, at the stage of zygotes and 2-cell embryos. The percentage of zygotes isolated after 3,4 and 7 days of L-Cn maternal treatment was reduced by $13(P=0.0163)$, $19(P=0.0157)$ and $31 \%(P<0.0001)$, respectively, as compared with controls, while the 5-day treatment did not seem to significantly affect zygote development (Fig. 4A). At the stage of 2-cell embryos, only the 7-day treatment affected embryo growth and reduced the percentage of normal embryos by $24 \% \quad(P=0.0429)$ 

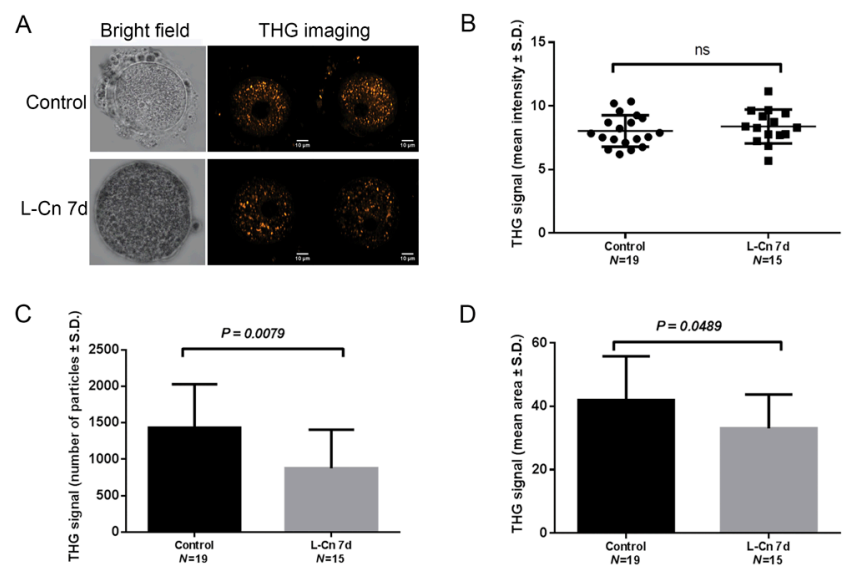

E

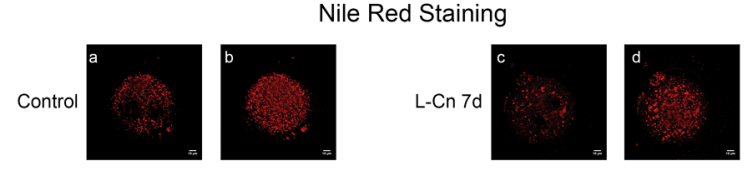

Figure 3 In vivo effect of L-Cn on zygote morphology. Superovulated females received a 7 -day treatment of $2.5 \mathrm{mg} /$ day L-Cn. Zygotes were isolated $18 \mathrm{~h}$ post-hCG injection and processed to THG imaging (A). Quantification of THG signaling included calculation of signal mean intensity (B), number of particles (C) and particle mean area (D). N represents the number of embryos used ( \pm S.D.). $P$ values were calculated using the GraphPad software statistical analysis tools (unpaired $t$-test). The experiments have been repeated at least five times. Zygotes were also stained with Nile red and visualized using confocal microscopy (E). A central section (Ea and Ec) and the maximal projection of all serial sections (Eb and $\mathrm{Ed}$ ) are shown.

as compared with controls (Fig. 4B). Thus, it seemed that after the 3-, 4- or 5-day L-Cn treatment, if zygotes survive, they can proceed to development. Indeed, mice could give birth to healthy offspring after the treatment of 3, 4 or 5 days with L-Cn (Fig. 4C). However, after the 7-day L-Cn treatment, mice never succeeded to give birth $(N>10)$. Following embryo development in this case, it was observed that on day 2 of pregnancy $(72 \mathrm{~h}$ post-hCG administration), only $22 \%$ of the embryos could reach the 8 -cell stage (Fig. 4D), while on day 3 of pregnancy, only $4 \%$ of the embryos could reach the stage of blastocyst (Fig. 4E). These results could indeed explain pregnancy failure in the 7-day L-Cn-treated females.

\section{Ovarian histology upon L-Cn treatment}

In order to evaluate oocyte quality, ovaries from day 0 of pregnancy from control and L-Cn-treated mice were collected, measured and processed for histological examination. The size of ovaries was estimated by calculating the mean total area of 10 central ovarian sections using the imagel software (Fig. 5A). The 7- and 5-day L-Cn treatment showed significantly bigger ovaries as compared with controls $(P=0.009$ and $P=0.039$, respectively) probably due to an inflammatory state manifested after
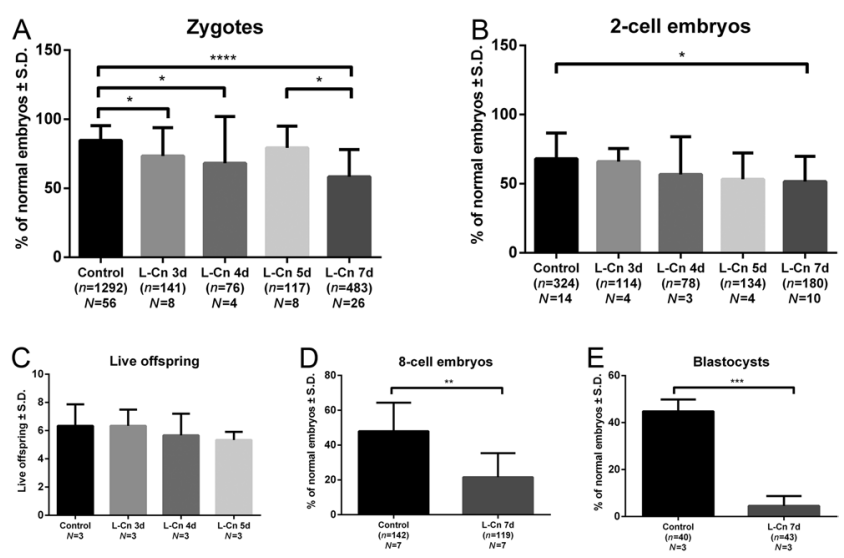

Figure 4 Effect of in vivo administration of L-Cn on embryo development. Superovulated female mice received daily injections of $2.5 \mathrm{mg}$ L-Cn for 3, 4, 5 and 7 days, caged with males and zygotes, 2-cell stage embryos, 8-cell stage embryos and blastocysts were isolated 18, 44, 72 or $96 \mathrm{~h}$ post-hCG injection, respectively. Live offspring were counted on the day of their birth. The percent of normal zygotes (A), 2-cell stage embryos (B), live offspring (C), 8-cell stage embryos (D) or blastocysts (E) was estimated. In all cases, $N$ represents the number of mice used in each case and $n$ represents the corresponding number of embryos tested. The results represent the mean of the embryos tested \pm s.D. $P$ values were calculated using the GraphPad software statistical analysis tools (unpaired $t$-test).

$* P<0.05, * * P<0.01, * * * P<0.001, * * * * P<0.0001$.

L-Cn administration (Fig. 5A). Ovarian histology could explain the defective embryo development observed in the 7-day L-Cn-treated females. Thus, control ovaries showed increased numbers of follicles and less corpora luteus. Despite cycle synchronization, in the ovaries of animals treated with L-Cn for 7 days, all stages of follicles could be observed, many of them positioned at the central part of the ovary, while ovarian cortex and medulla were showing abnormal organization and increased numbers of corpora luteus (Fig. 5B). Such observation is in agreement with the reduced percentage of zygotes obtained after the 7-day L-Cn treatment (Fig. 4A). Since corpus luteum ensures progesterone production during the early stages of pregnancy, increased progesterone levels would be expected in the maternal serum. Indeed, L-Cn-treated pregnant mice showed statistically significant higher progesterone levels (62\% of increase) as compared with control pregnant females (Fig. 5C). These results indicate that corpora luteus in the 7-day L-Cn-treated animals could be an additional source for serum progesterone.

\section{Maternal systemic immunity upon L-Cn treatment}

Pregnancy initially requires a maternal immunostimulatory environment to support early embryo growth, which at later stages will establish immunosuppressive mechanisms to ensure fetalmaternal homeostasis. The results so far indicated 

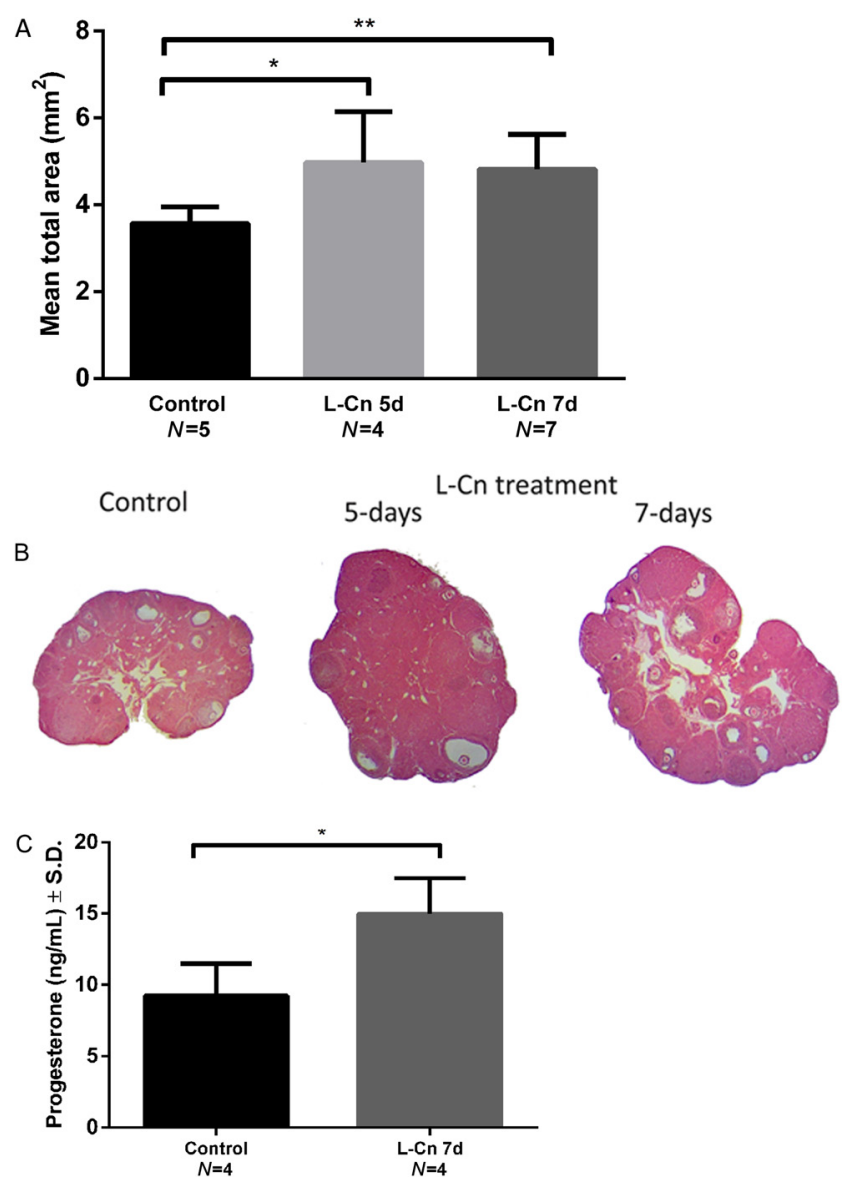

Figure 5 Effect of in vivo L-Cn treatment on ovarian histology. Superovulated female mice received daily injections of $2.5 \mathrm{mg}$ L-Cn for 5 or 7 days, caged with males and ovaries were isolated $18 \mathrm{~h}$ post-hCG injection on day 0 of pregnancy. Ovarian size was estimated by calculating the mean total area of 10 central ovarian sections using the imageJ software (A). Paraffin sections of $7 \mu \mathrm{m}$ were stained with hematoxylin-eosin (B). A central section of ovaries from control and L-Cn-treated mice (5 or 7 days of treatment) is shown. Progesterone levels were estimated by ELISA in the mouse serum (C). $N$ represents the number of ovaries tested from different animals (A) or the number of mice tested (C). The results represent the mean values of the ovaries or mice tested \pm S.D. $P$ values were calculated using the GraphPad software statistical analysis tools (unpaired $t$-test). ${ }^{*} P<0.05,{ }^{*} P P<0.01$.

that the L-Cn treatment affects ovarian structure, oocyte maturation as well as embryo development, which does not reach the stage to implant. In order to evaluate whether maternal systemic immunity could assist pregnancy failure, the effect of L-Cn treatment on immune spleen marker expression in spleen and growth factor production in serum was examined.

To this extend, spleens were isolated from L-Cntreated superovulated mice on day 0 of pregnancy (day of vaginal plug) and processed to immunofluorescence staining followed by flow cytometry analysis. The results showed that only the 7-day L-Cn treatment increased in a statistically significant manner the percent of CD19$(P=0.0177), C D 25-(P=0.0010), C D 11 b-(P=0.0004)$
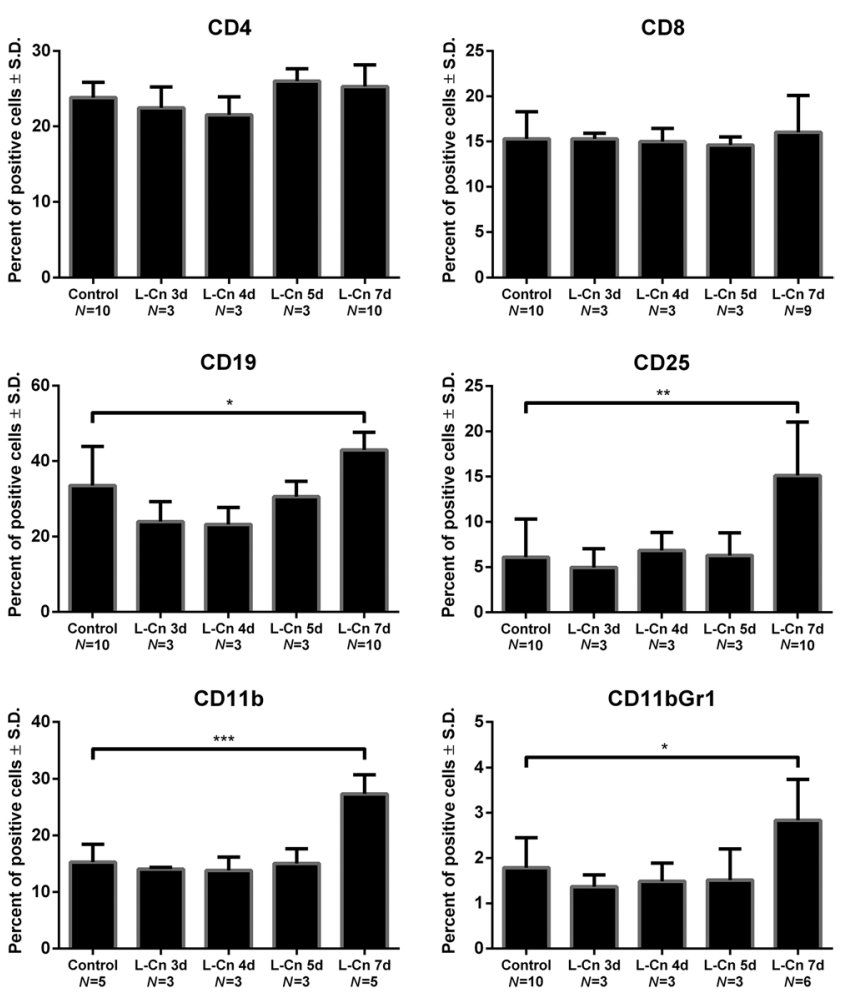

Figure 6 Effect of in vivo L-Cn treatment on maternal spleen immune cell populations. Superovulated female mice received daily injections of $2.5 \mathrm{mg} \mathrm{L-Cn}$ for 3, 4, 5 and 7 days, caged with males and spleens were isolated on day 0 of pregnancy. Spleen cells were submitted to immunofluorescence experiments and the percent of positive cells were evaluated using flow cytometry analysis. $\mathrm{N}$ represents the number of mice used in each case. The results represent the mean of at least three experiments ( \pm S.D.). $* P<0.05,{ }^{* *} P<0.01,{ }^{* * *} P<0.001$.

and CD11bGr1- $(P=0.0183)$ positive cell populations (Fig. 6). Thus, the inflammatory state of the mother could be mediated by the increased levels of macrophages in spleen, which is in accordance with previous results (Dionyssopoulou et al. 2005). In addition, the increase in the CD25 and CD11bGr1 (myeloidderived suppressor cells, MDSCs) cell population favors an immunosuppressive state to the mother, which opposes the immunostimulatory necessity at this early stage of pregnancy. These results are in accordance to the pregnancy failure observed after the 7-day L-Cn treatment stated in the previous section.

Concentrating on the 7-day L-Cn treatment, it was then inquired whether the observed suppression persisted for the next preimplantation stages. Thus, it was shown that the CD11- and CD25-positive cells reached control levels on days 1 and 2 of pregnancy (Fig. 7). MDSCs, however, did not follow control levels on day 2 of pregnancy. In this case, control mice showed a significant increase in MDSC levels $(P<0.0001)$ between days 0 and 2 of pregnancy), which could probably ensure the development of the required immunosuppressive state (Nair et al. 2015, Zhao et al. 2016), while L-Cn-treated mice failed to further increase these levels $(P=0.018$, 

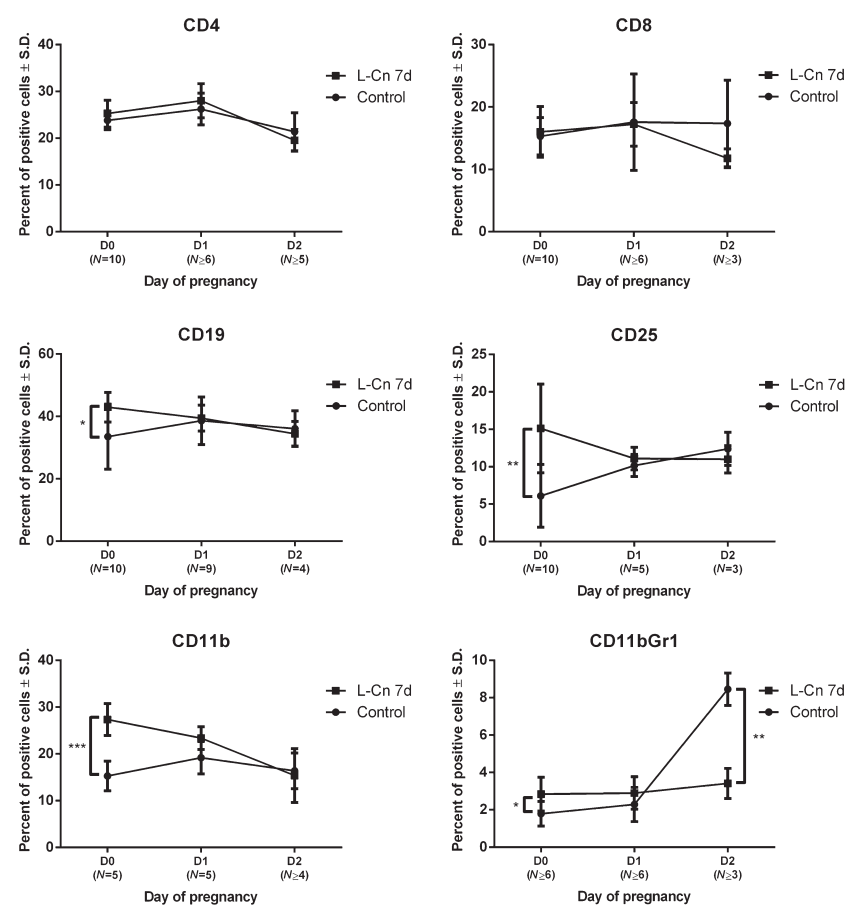

Figure 7 Effect of in vivo L-Cn treatment on maternal spleen immune cell populations. Superovulated female mice received daily injections of $2.5 \mathrm{mg}$ L-Cn for 7 days, caged with males and spleens were isolated on days 0,1 and 2 of pregnancy. Spleen cells were submitted to immunofluorescence experiments and the percent of positive cells were evaluated using flow cytometry analysis. $N$ represents the number of mice used in each case. The results represent the mean of at least three experiments ( \pm S.D.). ${ }^{*} P<0.05,{ }^{* *} P<0.01,{ }^{* * *} P<0.001$.

as compared with control levels). Taken together these results indicate that indeed the maternal systemic milieu could not sufficiently support embryo growth.

\section{Discussion}

Administration of L-Cn to female mice has been previously shown to induce an endometriosis-like state consisting of increased inflammatory cytokines in serum and peritoneal fluid, and accumulation of immune cells in the peritoneal cavity and uterine walls, which correlated to infertility (Dionyssopoulou et al. 2005). Taking advantage of nonlinear microscopy, which has allowed quantification of preimplantation embryo health (Kyvelidou et al. 2011), the present work focused on the infertility state of L-Cn-treated females and attempted to locate embryonic and ovarian defects leading to pregnancy failure. Comparing different doses of L-Cn, it was shown that administration of $2.5 \mathrm{mg}$ for 7 days affected preimplantation embryo viability, by significantly reducing the percent of recovered blastocysts on day 3 of pregnancy, while disorganizing ovarian histology accompanied by abnormal immunostimulatory vs immunosuppressive balance to the mother, correlated to the inability of pregnancy support.
Initial studies as to the effect of L-Cn on preimplantation embryo development in vitro showed that indeed, $400 \mu \mathrm{M}$ of $\mathrm{L}-\mathrm{Cn}$ decreased the rate of zygotes to 2-cell embryo differentiation, while also decreasing THG signal levels, which was previously shown correlated to embryo health (Kyvelidou et al. 2011). Third harmonic generation visualizes essentially LBs and to a lesser degree mitochondria (Débarre et al. 2006, Hsieh et al. 2008, Watanabe et al. 2010). In the preimplantation embryos studied herein, nile red could co-localize with THG signal and, therefore, THG signal quantification was proportional to the LB content of the cells. Thus, $\mathrm{L}-\mathrm{Cn}$, whose role is to deliver long-chain fatty acids to mitochondria for catabolism, was shown to decrease LB in the cells, which indirectly could indicate an increase in the energetic status of the cells. Indeed, it has been suggested that L-Cn ameliorates maturation of mouse oocytes and early embryo development (Zare et al. 2015), while decreasing DNA damage and improving the in vitro blastocyst development rate in mouse embryos (Abdelrazik et al. 2009). It could, therefore, be hypothesized that in the presence of L-Cn, zygotes with limited energetic reserves could not proceed to development and thus only healthy embryos could reach the stage of 2-cells. Yet, further experimentation is needed to test such hypothesis.

The in vivo administration of L-Cn, however, results in more complicated effects, since L-Cn can affect every single cell type of the organism producing various responses (Dionyssopoulou et al. 2011). Among others, L-Cn has been shown to increase the arachidonic acidinduced production of PGE1 and PGE2 by macrophages (Athanassakis et al. 2003), reduce IL-2 production in response to specific antigenic stimulus (Athanassakis et al. 2001) or induce an endometriosis-like state to female mice and infertility (Dionyssopoulou et al. 2005).

Injection of L-Cn to mice is preferred over oral administration since it has been shown to be more effective (Lawanlakkana et al. 2012) and ensures inoculation of the exact desired dose of the agent. The applied L-Cn dosage has been previously shown to become acylated and consequently enter the various biochemical pathways (Dionyssopoulou et al. 2005).

Following the L-Cn-induced endometriosis-like state, it was shown by THG imaging analysis that zygotes isolated from females having received the 7-day L-Cn treatment contained significantly lower numbers of LBs with reduced mean area, displaying coagulations and unusual depositions.

Since the 7-day L-Cn treatment leads to infertility, it was then attempted to define the lowest L-Cn dose affecting embryo development. Thus, female mice were treated for 3-7 days with L-Cn and embryos were collected at the stage of zygote or the 2-cell embryo. In order to ensure estrus, in all cases, mice were superovulated. Although the numbers of normal zygotes was affected after 3, 4 and 7 days of treatment, the 2 -cell stage embryos were only 
affected after the 7-day treatment. Thus, it seemed that if zygotes survive after the 3, 4 or 5 days of L-Cn treatment, they can proceed to development. The 7-day treatment, however, seemed to irreversibly damage embryo growth. Indeed, on day 3 of pregnancy, only $5 \%$ of the embryos could reach the blastocyst level, explaining thus the inability to pregnancy progression. However, it has to be noted that the effect of L-Cn was reversible and females recovered fertility 3 months after treatment cessation.

Ovarian histology correlated with the poor oocyte quality observed after the 7-day L-Cn treatment. Ovaries were bigger in size, with abnormal organization in cortex and medulla, increased numbers of corpora luteus and despite cycle synchronization all stages of follicles were observed, positioned centrally to the ovary. These observations were correlated with increased progesterone in the serum of 7-day L-Cntreated pregnant females.

Systemic maternal immunity was evaluated through specific marker expression in the spleen. It has to be noted that successful pregnancy begins with a maternal immunostimulatory state to support embryo growth, followed by an immunosuppressive state to equilibrate embryo growth against the maternal organism (Athanassakis \& Wegmann 1986). The 7-day treatment was the only one showing significant increase in CD19- (B cells), CD25- (Tregs), CD11b(macrophages) and CD11bGr1- (MDSCs) positive cells on day 0 of pregnancy in spleen. The increased numbers of macrophages would be indicative of an inflammatory condition, while the increase in Tregs and MDSCs would be indicative of an immunosuppressive state, which opposes the necessity of immunostimulation for successful pregnancy initiation. To this extend, maternal serum collected after the 7-day L-Cn treatment showed to contain increased levels of the immunosuppressive cytokine IL-10 $(P<0.05$ as compared with controls; data not shown), which has been found increased in women with reproductive failure and has been suggested to participate in early pregnancy (Mahdi 2011). By the same token, increased levels of progesterone could be detected in the 7-day L-Cn-treated pregnant mice, which could further fortify the immunosuppressive state of the mother early in pregnancy (Xu et al. 2011). Thus, the systemic immunity could not favor embryo development. However, following these markers on days 1 and 2 of pregnancy, it was shown that except from MDSCs, all other cell populations conformed to control levels. MDSCs, although increased on day 0 of pregnancy as compared with controls, their numbers did not increase thereafter to support the immunosuppressive state, necessary for further embryo development.

In conclusion, the 7-day treatment, which consisted of 7 daily injections of $2.5 \mathrm{mg}$ L-Cn, altered the LB of zygotes leading to abnormal embryo development, almost unable to reach the blastocyst stage and, therefore, to implant. Such embryo inefficiency seemed to originate from low oocyte quality due to ovarian dysfunction as well as disequilibrium of systemic maternal immunity. Thus, the herein described L-Cn-induced infertility could represent a cause of unexplained so far infertility. Considering that L-Cn supplements many formulas for weight loss, muscle and bone mass building, brain function etc., its use may account for inability to pregnancy.

\section{Declaration of interest}

The authors declare that there is no conflict of interest that could be perceived as prejudicing the impartiality of the research reported or any financial or other potential conflict of interest. It has to be declared that the authors I Athanassakis, G Filippidis, C Kyvelidou and G Tserevelakis have patented THG imaging technology in preimplantation embryo quality evaluation in a patent (OBI 20110100030) entitled 'Use of non-linear imaging in pre-implantation embryo quality evaluation for successful pregnancy'.

\section{Funding}

This research did not receive any specific grant from any funding agency in the public, commercial or not-for-profit sector.

\section{Acknowledgements}

The authors would like to thank Prof Tavernarakis and Prof Tokatlidis $\mathrm{N}$ for Nile red and mitotracker, respectively, and Prof Chalepakis for allowing the use of the equipment in his laboratory for performing ovarian histological analysis.

\section{References}

Abdelrazik H, Sharma R, Mahfouz R \& Agarwal A 2009 L-carnitine decreases DNA damage and improves the in vitro blastocyst development rate in mouse embryos. Fertility and Sterility 91 589-596. (doi:10.1016/j. fertnstert.2007.11.067)

Athanassakis I and Wegmann TG 1986 Immunostimulation and the promotion of fertility. In Immunological Approaches to Contraception and Promotion of Fertility, pp 407-415. Ed GP Talwar. Boston, MA, USA: Springer US.

Athanassakis I, Mouratidou M, Sakka P, Evangeliou A, Spilioti M \& Vassiliadis S 2001 L-carnitine modifies the humoral immune response in mice after in vitro or in vivo treatment. International Immunopharmacology 1 1813-1822. (doi:10.1016/S1567-5769(01)00105-9)

Athanassakis I, Dionyssopoulou E, Papanikou S, Evangeliou A \& Vassiliadis S 2003 Early events of the exogenously provided L-Carnitine in murine macrophages, $T$ - and B-lymphocytes: modulation of prostaglandin E1 and E2 production in response to arachidonic acid. Journal of Nutritional Biochemistry 14 350-357. (doi:10.1016/s0955-2863(03)00058-5)

Barad Y, Eisenberg H, Horowitz M \& Silberberg Y 1997 Nonlinear scanning laser microscopy by third harmonic generation. Applied Physics Letters 70 922. (doi:10.1063/1.118442)

Brass EP 2004 Carnitine and sports medicine: use or abuse? Annals of the New York Academy of Sciences 1033 67-78. (doi:10.1196/ annals.1320.006)

Debarre D \& Beaurepaire E 2007 Quantitative characterization of biological liquids for third-harmonic generation microscopy. Biophysical Journal 92 603-612. (doi:10.1529/biophysj.106.094946) 
Débarre D, Supatto W, Pena A-M, Fabre A, Tordjmann T, Combettes L, Schanne-Klein M-C \& Beaurepaire E 2006 Imaging lipid bodies in cells and tissues using third-harmonic generation microscopy. Nature Methods 3 47-53. (doi:10.1038/nmeth813)

Dionyssopoulou E, Vassiliadis S, Evangeliou A, Koumantakis EE \& Athanassakis I 2005 Constitutive or induced elevated levels of L-carnitine correlate with the cytokine and cellular profile of endometriosis. Journal of Reproductive Immunology 65 159-170. (doi:10.1016/j.jri.2004.12.002)

Dionyssopoulou E, Anagnostaki E, Tselekidou A, Vassiliadis S \& Athanassakis I 2011 Establishment or aggravation of endometriosis by L-carnitine: the role of PGE1 and PGE2 in the endometriosisinduction process. In New Developments in Endometriosis, p 632. Eds I Matalliotakis \& A Arici. CreateSpace Independent Publishing Platform.

Famularo G, Marticardi F, Nucera E, Santini G \& De Simone C 1977 Carnitine deficiency: primary and secondary syndromes. In Carnitine Today, pp 120-161. Eds C De Simone \& G Famularo. New York, NY, USA: Landes Bioscience and Chapman \& Hall.

Hsieh C-S, Chen S-U, Lee Y-W, Yang Y-S \& Sun C-K 2008 Higher harmonic generation microscopy of in vitro cultured mammal oocytes and embryos. Optics Express 16 11574-11588.

Karpati G, Carpenter S, Engel AG, Watters G, Allen J, Rothman S, Klassen G \& Mamer OA 1975 The syndrome of systemic carnitine deficiency. Clinical, morphologic, biochemical, and pathophysiologic features. Neurology 25 16-24. (doi:10.1212/wnl.25.1.16)

Kito S, Hayao T, Noguchi-Kawasaki Y, Ohta Y, Hideki U \& Tateno S 2004 Improved in vitro fertilization and development by use of modified human tubal fluid and applicability of pronucleate embryos for cryopreservation by rapid freezing in inbred mice. Comparative Medicine 54 564-570.

Kyvelidou C, Tserevelakis GJ, Filippidis G, Ranella A, Kleovoulou A, Fotakis C \& Athanassakis I 2011 Following the course of pre-implantation embryo patterning by non-linear microscopy. Journal of Structural Biology 176 379-386. (doi:10.1016/j.jsb.2011.09.007)

Lawanlakkana P, Saraithong S, Suwantemee C \& Pitiseree A 2012 Comparing the effect between oral and injection form of carnitine on skin flap survival in rats. Journal of the Medical Association of Thailand 95 S157-161.

Mahdi BM 2011 Role of some cytokines on reproduction. Middle East Fertility Society Journal 16 220-223. (doi:10.1016/j.mefs.2011.03.002)
Michal G 1999 Arachidonate metabolism and eicosanoids. In Biochemical Pathways: An Atlas of Biochemistry and Molecular Biology, 1st ed, p 288. New York, NY, USA: John Wiley \& Sons, Inc. (doi:10.1002/9781118657072)

Nair RR, Sinha P, Khanna A \& Singh K 2015 Reduced myeloid-derived suppressor cells in the blood and endometrium is associated with early miscarriage. American Journal of Reproductive Immunology 73 479-486. (doi:10.1111/aji.12351)

Ng CM, Blackman MR, Wang C \& Swerdloff RS 2004 The role of carnitine in the male reproductive system. Annals of the New York Academy of Sciences 1033 177-188. (doi:10.1196/annals.1320.017)

Watanabe T, Thayil A, Jesacher A, Grieve K, Debarre D, Wilson T, Booth M \& Srinivas S 2010 Characterisation of the dynamic behaviour of lipid droplets in the early mouse embryo using adaptive harmonic generation microscopy. BMC Cell Biology 11 38. (doi:10.1186/14712121-11-38)

Xu Y, He H, Li C, Shi Y, Wang Q, Li W \& Song W 2011 Immunosuppressive effect of progesterone on dendritic cells in mice. Journal of Reproductive Immunology 91 17-23. (doi:10.1016/j.jri.2011.06.101)

Zare Z, Masteri Farahani R, Salehi M, Piryaei A, Ghaffari Novin M, Fadaei Fathabadi F, Mohammadi M \& Dehghani-Mohammadabadi $M$ 2015 Effect of L-carnitine supplementation on maturation and early embryo development of immature mouse oocytes selected by brilliant cresyle blue staining. Journal of Assisted Reproduction and Genetics 32 635-643. (doi:10.1007/s10815-015-0430-5)

Zhao A-M, Xu H-J, Kang X-M, Zhao A-M \& Lu L-M 2016 New insights into myeloid-derived suppressor cells and their roles in feto-maternal immune cross-talk. Journal of Reproductive Immunology 113 35-41. (doi:10.1016/j.jri.2015.11.001)

Received 2 April 2016

First decision 30 April 2016

Revised manuscript received 28 May 2016

Accepted 7 July 2016 